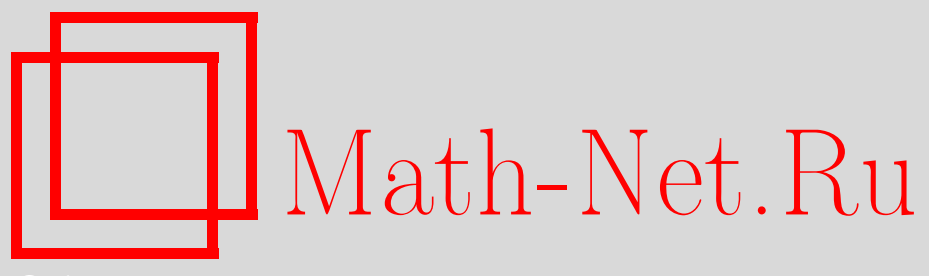

С. И. Адян, Ф. Груневальд, Й. Меннике, А. Л. Таламбуца, Автоморфизмы свободных групп и группы классов отображений двумерных поверхностей, Матем. заметки, 2007, том 81, выпуск 2, 163-173

DOI: https://doi.org/10.4213/mzm3544

Использование Общероссийского математического портала Math-Net.Ru подразумевает, что вы прочитали и согласны с пользовательским соглашением http://www . mathnet.ru/rus/agreement

Параметры загрузки:

IP: 35.173 .219 .149

26 апреля 2023 г., 14:21:13

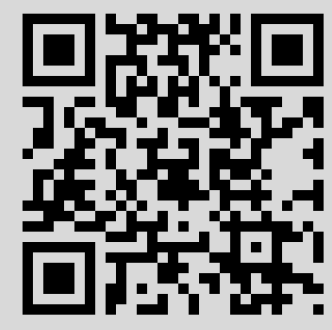


Том 81 выпуск 2 февраль 2007

УДК 512.54

\title{
Автоморфизмы свободных групп и группы классов отображений двумерных поверхностей
}

\author{
С. И. Адян, Ф. Груневальд, Й. Меннике, А. Л. Таламбуца
}

Пусть $N$ есть стабилизатор слова $w=s_{1} t_{1} s_{1}^{-1} t_{1}^{-1} \ldots s_{g} t_{g} s_{g}^{-1} t_{g}^{-1}$ в группе автоморфизмов $\operatorname{Aut}\left(F_{2 g}\right)$ свободной группы с порождающими $\left\{s_{i}, t_{i}\right\}_{i=1, \ldots, g}$. Фундаментальная группа $\pi_{1}\left(\Sigma_{g}\right)$ двумерной компактной ориентируемой замкнутой поверхности рода $g$ в порождающих $\left\{s_{i}, t_{i}\right\}$ задается определяющим соотношением $w=1$. В работе найдены элементы $S_{i}, T_{i} \in N$, которые в $\operatorname{Aut}\left(\pi_{1}\left(\Sigma_{g}\right)\right)$ задают сопряжение с помощью порождающих $s_{i}, t_{i}$. Они вместе с элементом $\alpha \in N$, реализующим сопряжение с помощью $w$, порождают ядро естественного эпиморфизма группы $N$ на группу классов отображений $M_{g, 0}=$ $\operatorname{Aut}\left(\pi_{1}\left(\Sigma_{g}\right)\right) / \operatorname{Inn}\left(\pi_{1}\left(\Sigma_{g}\right)\right)$. Найдена система определяющих соотношений этого ядра в порождающих $S_{1}, \ldots, S_{g}, T_{1}, \ldots, T_{g}, \alpha$. Кроме того, в $N$ найдена изоморфная группе кос $B_{g}$ на $g$ нитях подгруппа, которая в результате абелизации свободной группы $F_{2 g}$ отображается на подгруппу группы Вайля $\operatorname{Sp}(2 g, \mathbb{Z})$, состоящую из матриц, которые содержат только 0 и 1.

Библиография: 9 названий.

Группа классов отображений двумерной компактной ориентированной поверхности данного рода есть группа ее гомеоморфизмов на себя по модулю гомотопий. Она рассматривалась многими авторами. Систему порождающих для нее впервые нашел Дэн [1]; эти порождающие теперь называются скручиваниями Дэна (Dehn twists). Позже их уточняли Ликориш [2] и Хамфри [3]. Важный шаг на пути описания системы определяющих соотношений был сделан в работе Хэтчера и Тёрстона [4]. Используя идеи этих авторов, Вайнрыб в работе [5] дал описание системы определяющих соотношений групп классов отображений.

Группу классов отображений, сохраняющих ориентацию двумерной поверхности рода $g$ с $n$ выколотыми точками, будем обозначать через $M_{g, n}$. В настоящей работе мы будем рассматривать группы $M_{g, 0}, M_{g, 1}$ в терминах автоморфизмов фундаментальной группы рассматриваемой поверхности при $g \geqslant 2$.

Пусть нам дана ориентированная двумерная компактная поверхность $\Sigma_{g}$ рода $g \geqslant 2$ и на ней указаны следующие ориентированные простые замкнутые кривые (см. рис. 1):

$$
u_{i}, y_{i}, \quad i=1,2, \ldots, g, \quad x_{j}, \quad j=2,3, \ldots, g .
$$

Работа выполнена при поддержке Российского фонда фндаментальных исследований, фонда DFG и прогрвммы "Ведущие научные школы" (грант № НШ-5351.2006.1) 


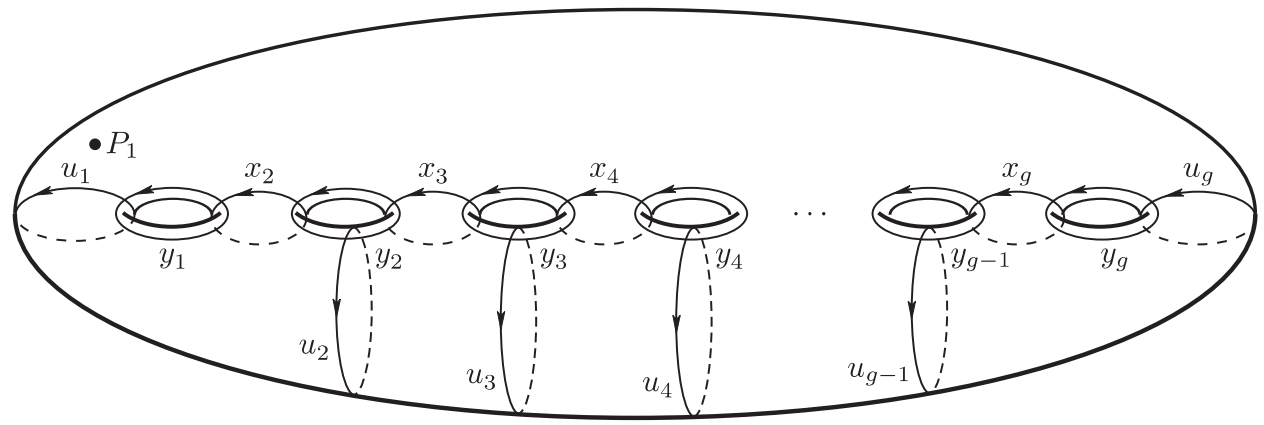

Рис. 1. Базовые кривые для порождающих скручиваний Дэна

Для каждой из этих кривых определен связанный с ней гомеоморфизм поверхности, называемый скручиванием или твистом Дэна. Твист Дэна, соответствующий простой ориентированной кривой, определяется следующим образом. Вырезаем из поверхности небольшой цилиндр вдоль этой кривой так, что кривая является средней линией этого цилиндра. Нижней окружностью цилиндра назовем ту окружность, что находится слева от ориентированной средней линии. Скручиваем верхнюю окружность на $360^{\circ}$ в направлении ориентации средней линии, оставляя неподвижной нижнюю окружность. Затем вставляем полученный цилиндр обратно в поверхность. В результате получим гомеоморфное отображение исходной поверхности на себя, называемое скручиванием Дэна, связанным с заданной ориентированной кривой. Скручивания Дэна, связанные с кривыми $u_{i}, x_{i}, y_{i}$ на рис. 1 , мы будем также обозначать через $u_{i}, x_{i}, y_{i}$. При рассмотрении группы $M_{g, 1}$ выколотую точку $P_{1}$ выберем в верхнем левом углу, как указано на рис. 1.

Следующая лемма хорошо известна.

ЛЕмма 1 (Дэн, 1938; Ликориш, 1964, 1966). Скручивания Дэна

$$
u_{i}, y_{i}, x_{j}, \quad i=1,2, \ldots, g, \quad j=2,3, \ldots, g,
$$

порождают группу классов отображений $M_{g, n}$ nри $n=0,1$.

Фундаментальная группа $\pi_{1}\left(\Sigma_{g}\right)$ порождается элементами $s_{i}, t_{i}, i=1,2, \ldots, g$, кривые для которых указаны на рис. 2. При этом базовая точка $B$ располагается в верхнем левом углу повехности.

Обозначим

$$
k_{i}=s_{i} t_{i} s_{i}^{-1} t_{i}^{-1}, \quad i=1,2, \ldots, g, \quad w=k_{1} k_{2} \ldots k_{g} .
$$

Каждому гомеоморфизму поверхности $\Sigma_{g}$ естественным образом сопоставляется автоморфизм фундаментальной группы, имеющей задание

$$
\pi_{1}\left(\Sigma_{g}\right)=\left\langle s_{i}, t_{i} \mid w=1\right\rangle
$$

(более подробно об этом см. [6]).

Укажем, как действуют скручивания Дэна (2) на порождающие фундаментальной группы $\pi_{1}\left(\Sigma_{g}\right)$. При этом мы будем для краткости записывать порождающие 


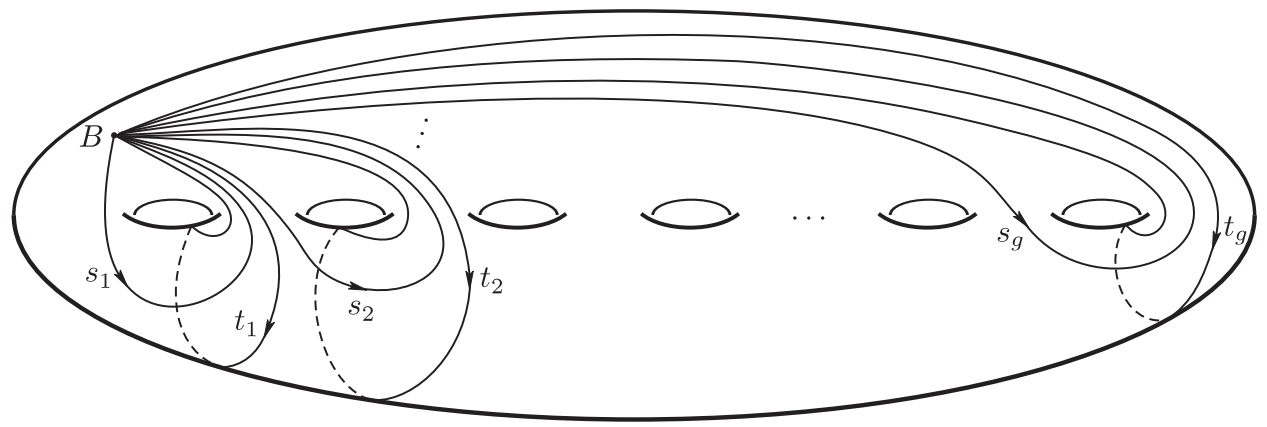

Рис. 2. Порождающие фундаментальной группы

$s_{i}^{-1}$ и $t_{i}^{-1}$ через $s_{i}^{\prime}$ и $t_{i}^{\prime}$ и не будем указывать преобразования тех порождающих $s_{j}$, $t_{j}$, которые переводятся в себя. Аналогичное сокращение $x^{\prime}$ вместо $x^{-1}$ мы будем использовать и для обозначения обратных автоморфизмов. Имеем

$$
\begin{aligned}
& u_{i}=\left\{s_{i}-\rightarrow s_{i} t_{i}^{\prime}, \quad i=1,2, \ldots, g,\right. \\
& y_{i}=\left\{t_{i} \rightarrow t_{i} s_{i},\right. \\
& x_{j}=\left\{\begin{array}{c}
s_{j-1}-\rightarrow s_{j-1} t_{j-1}^{\prime} s_{j} t_{j} s_{j}^{\prime}, \\
t_{j-1}-\rightarrow s_{j} t_{j}^{\prime} s_{j}^{\prime} t_{j-1} s_{j} t_{j} s_{j}^{\prime}, \quad j=2,3, \ldots, g . \\
s_{j--\rightarrow} s_{j} t_{j}^{\prime} s_{j}^{\prime} t_{j-1} s_{j},
\end{array}\right.
\end{aligned}
$$

Укажем, как действуют обратные отображения $x_{j}^{\prime}$ :

$$
x_{j}^{\prime}=\left\{\begin{aligned}
s_{j-1} & -\rightarrow s_{j-1} s_{j} t_{j}^{\prime} s_{j}^{\prime} t_{j-1}, \\
t_{j-1} & -\rightarrow t_{j-1}^{\prime} s_{j} t_{j} s_{j}^{\prime} t_{j-1} s_{j} t_{j}^{\prime} s_{j}^{\prime} t_{j-1}, \quad j=2,3, \ldots, g . \\
s_{j} & -\rightarrow t_{j-1}^{\prime} s_{j} t_{j},
\end{aligned}\right.
$$

Действие обратных отображений $u_{j}^{\prime}$ и $y_{j}^{\prime}$ очевидно.

Отображения (4) можно рассматривать как автоморфизмы свободной группы $F_{2 g}$ с порождающими

$$
s_{1}, t_{1}, s_{2}, t_{2}, \ldots, s_{g}, t_{g}
$$

Через $N$ обозначим стабилизатор слова $w$ в группе автоморфизмов $\operatorname{Aut}\left(F_{2 g}\right)$. В данной работе мы используем следующий порядок композиции автоморфизмов:

$$
\left(\tau_{1} \tau_{2}\right)\left(s_{i}, t_{i}\right)=\tau_{1}\left(\tau_{2}\left(s_{i}, t_{i}\right)\right)
$$

Легко проверить, что все автоморфизмы (4), рассматриваемые на свободной группе $F_{2 g}$, оставляют неподвижным слово $w$, т.е. имеем

$$
u_{i}, y_{i}, x_{j} \in N=\operatorname{Stab}(w), \quad i=1,2, \ldots, g, \quad j=2,3, \ldots, g .
$$

Пусть $\mathrm{Aut}^{+}\left(F_{2 g}\right)$ есть группа всех автоморфизмов, которые отображают слово $w$ в сопряженное ему слово. Известно, что группа классов отображений $M_{g, 1}$ изоморфна 
факторгруппе группы $\mathrm{Aut}^{+}\left(F_{2 g}\right)$ по подгруппе внутренних автоморфизмов $\operatorname{Inn}\left(F_{2 g}\right)$ (cM. [7; c. 184]):

$$
M_{g, 1}=\operatorname{Aut}^{+}\left(F_{2 g}\right) / \operatorname{Inn}\left(F_{2 g}\right) .
$$

Согласно теореме Дэна-Нильсена (см. [6; теорема 5.15.3]) и замечанию к теореме N10 (см. [7; с. 186]) группу $M_{g, 0}$ можно алгебраически определить как

$$
M_{g, 0}=\operatorname{Aut}^{+}\left(\pi_{1}\left(\Sigma_{g}\right)\right) / \operatorname{Inn}\left(\pi_{1}\left(\Sigma_{g}\right)\right),
$$

где через $\mathrm{Aut}^{+}\left(\pi_{1}\right)$ обозначен образ группы $\mathrm{Aut}^{+}\left(F_{2 g}\right)$ при естественном отображении ее в группу $\operatorname{Aut}\left(\pi_{1}\right)$.

Через $\alpha$ обозначим внутренний автоморфизм группы $F_{2 g}$, переводящий любой элемент $x$ в $w^{-1} x w$, т.е.

$$
\alpha: x \rightarrow w^{-1} x w .
$$

Лемма 2. Группа $M_{g, 1}$ изоморфна факторгруппе $N /\langle\alpha\rangle$.

Доказательство. Так как $N \subset \operatorname{Aut}^{+}\left(F_{2 g}\right)$ и $N \cap \operatorname{Inn}\left(F_{2 g}\right)$ есть нормальная подгруппа группы $N$, то имеем естественное вложение факторгруппы $N /\left(N \cap \operatorname{Inn}\left(F_{2 g}\right)\right)$ в факторгруппу $\mathrm{Aut}^{+}\left(F_{2 g}\right) / \operatorname{Inn}\left(F_{2 g}\right)$. Это вложение есть изоморфизм, так как любой элемент $\gamma \in \operatorname{Aut}^{+}\left(F_{2 g}\right)$ эквивалентен по модулю $\operatorname{Inn}\left(F_{2 g}\right)$ некоторому элементу $\gamma_{1} \in N$. Нам остается доказать равенство $N \cap \operatorname{Inn}\left(F_{2 g}\right)=\langle\alpha\rangle$.

Если $\beta \in N \cap \operatorname{Inn}\left(F_{2 g}\right)$, то при некотором $T$ для любого $x \in F_{2 g}$ имеем $\beta(x)=$ $T x T^{-1}$. Тогда из $\beta \in N$ следует $T w T^{-1}=w$ в свободной группе, т.е. слова $T$ и $w$ коммутируют. Тогда они являются степенями одного и того же слова. Так как слово $w$ не равно в свободной группе никакой степени другого слова, то слово $T$ равно в свободной группе некоторой степени слова $w$, т.е. автоморфизм $\beta$ является некоторой степенью внутреннего автоморфизма $\alpha$. Лемма 2 доказана.

\section{ЛЕмма 3. Группа $N$ порождается автоморфизмами (4).}

ДокАЗАТЕЛЬСтво. В силу лемм 1 и 2 достаточно доказать, что автоморфизм $\alpha$ можно представить в $N$ как произведение автоморфизмов (4). Это вытекает из соотношения

$$
\alpha=\left(u_{1} y_{1} x_{2} y_{2} \ldots x_{g} y_{g} u_{g}^{2} y_{g} x_{g} \ldots y_{2} x_{2} y_{1} u_{1}\right)^{2},
$$

которое доказывается непосредственным вычислением правой части с использованием автоморфизмов (4).

Легко проверить, что следующие ниже автоморфизмы свободной группы $S_{i}$ и $T_{i}$ также принадлежат стабилизатору $N$, т.е. они оставляют неподвижным элемент $w$ :

$$
\begin{aligned}
& S_{1}=\left\{\begin{array}{l}
s_{1--\rightarrow s_{1}}, \\
t_{1}-\rightarrow w^{\prime} s_{1} t_{1} s_{1}^{\prime}, \\
s_{j}-\rightarrow w^{\prime} s_{1} s_{j} s_{1}^{\prime} w, \\
t_{j}-\rightarrow w^{\prime} s_{1} t_{j} s_{1}^{\prime} w,
\end{array}\right. \\
& T_{1}=\left\{\begin{array}{l}
s_{1}-\rightarrow w t_{1} s_{1} t_{1}^{\prime}, \\
t_{1}-\rightarrow t_{1}, \\
s_{j}-\rightarrow t_{1} w s_{j} w^{\prime} t_{1}^{\prime}, \\
t_{j}-\rightarrow t_{1} w t_{j} w^{\prime} t_{1}^{\prime},
\end{array}\right.
\end{aligned}
$$




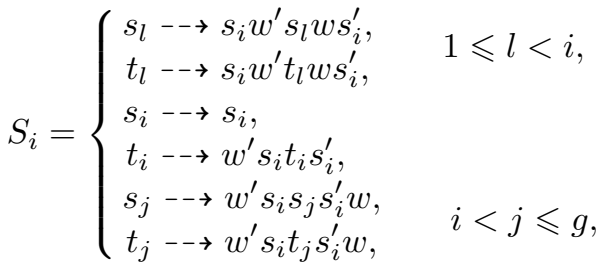

$$
\begin{aligned}
& T_{i}=\left\{\begin{array}{l}
s_{l} \rightarrow w t_{i} s_{l} t_{i}^{\prime} w^{\prime}, \quad 1 \leqslant l<i, \\
t_{l} \rightarrow w t_{i} t_{l} t_{i}^{\prime} w^{\prime}, \\
s_{i} \rightarrow w t_{i} s_{i} t_{i}^{\prime}, \\
t_{i} \rightarrow t_{i}, \\
s_{j} \rightarrow t_{i} w s_{j} w^{\prime} t_{i}^{\prime}, \quad 2 \leqslant i<j \leqslant g, \\
t_{j}-\rightarrow t_{i} w t_{j} w^{\prime} t_{i}^{\prime}, \quad
\end{array}\right.
\end{aligned}
$$

Для удобства читателя выпишем автоморфизмы, обратные к перечисленным выше:

$$
\begin{aligned}
& S_{1}^{\prime}=\left\{\begin{array}{l}
s_{1} \rightarrow s_{1}, \\
t_{1}-\rightarrow s_{1}^{\prime} w t_{1} s_{1}, \\
s_{j}-\rightarrow s_{1}^{\prime} w s_{j} w^{\prime} s_{1}, \\
t_{j}-\rightarrow s_{1} w t_{j} w^{\prime} s_{1},
\end{array} \quad 2 \leqslant j \leqslant g,\right. \\
& T_{1}^{\prime}=\left\{\begin{array}{l}
s_{1} \rightarrow t_{1}^{\prime} w^{\prime} s_{1} t_{1}, \\
t_{1}-\rightarrow t_{1}, \\
s_{j}-\rightarrow w^{\prime} t_{1}^{\prime} s_{j} t_{1} w, \\
t_{j} \rightarrow w^{\prime} t_{1}^{\prime} t_{j} t_{1} w,
\end{array}\right. \\
& S_{i}^{\prime}=\left\{\begin{array}{l}
s_{l} \rightarrow w s_{i}^{\prime} s_{l} s_{i} w^{\prime}, \quad 1 \leqslant l<i, \\
t_{l} \rightarrow w s_{i}^{\prime} t_{l} s_{i} w^{\prime}, \\
s_{i} \rightarrow s_{i}, \\
t_{i} \rightarrow s_{i}^{\prime} w t_{i} s_{i}, \\
s_{j} \rightarrow s_{i}^{\prime} w s_{j} w^{\prime} s_{i}, \quad i<j \leqslant g, \\
t_{j} \rightarrow s_{i}^{\prime} w t_{j} w^{\prime} s_{i},
\end{array}\right. \\
& T_{i}^{\prime}=\left\{\begin{array}{l}
s_{l} \rightarrow t_{i}^{\prime} w^{\prime} s_{l} w t_{i}, \quad 1 \leqslant l<i, \\
t_{l}-\rightarrow t_{i}^{\prime} w^{\prime} t_{l} w t_{i}, \\
s_{i}-\rightarrow t_{i}^{\prime} w^{\prime} s_{i} t_{i}, \\
t_{i}-\rightarrow t_{i}, \\
s_{j}-\rightarrow w^{\prime} t_{i}^{\prime} s_{j} t_{i} w, \quad 2 \leqslant i<j \leqslant g . \\
t_{j}-\rightarrow w^{\prime} t_{i}^{\prime} t_{j} t_{i} w,
\end{array}\right.
\end{aligned}
$$

Обозначим

$$
c=u_{1} y_{1} x_{2} y_{2} \ldots x_{g} y_{g} u_{g}^{2} y_{g} x_{g} \ldots y_{2} x_{2} y_{1} u_{1} .
$$

Последовательным вычислением соответствующих автоморфизмов легко доказывается, что автоморфизмы $S_{i}$ и $T_{i}$ выражаются через порождающие (4) следующим образом:

$$
\begin{array}{rlrl}
S_{1} & =u_{1} y_{1} u_{1} c u_{1} c^{\prime} u_{1}^{\prime} u_{1}^{\prime} y_{1}^{\prime} u_{1}^{\prime}, & \\
S_{i} & =x_{i} y_{i} y_{i-1} x_{i} S_{i-1} x_{i}^{\prime} y_{i-1}^{\prime} y_{i}^{\prime} x_{i}^{\prime}, & & 2 \leqslant i \leqslant g, \\
T_{j}=u_{j} y_{j} u_{j} S_{j}^{\prime} u_{j}^{\prime} y_{j}^{\prime} u_{j}^{\prime}, & & 1 \leqslant j \leqslant g .
\end{array}
$$


Очевидно, автоморфизмы $S_{i}$ и $T_{i}$ на группе $\pi_{1}\left(\Sigma_{g}\right)$ действуют как сопряжения с помощью порождающих $s_{i}$ и $t_{i}$ соответственно. Это означает, что они порождают всю группу $\operatorname{Inn}\left(\pi_{1}\left(\Sigma_{g}\right)\right)$, т.е. верна

ЛЕмма 4. Для любого элемента $\gamma \in \operatorname{Inn}\left(\pi_{1}\left(\Sigma_{g}\right)\right)$ можно указать такой свободный автоморфизм $f\left(S_{1}, T_{1}, \ldots, S_{g}, T_{g}\right) \in N$, который на группе $\pi_{1}\left(\Sigma_{g}\right)$ действует как $\gamma$.

В работе [8] Цишанг впервые указал принадлежащие стабилизатору $N$ автоморфизмы, образы которых в $\operatorname{Aut}\left(\pi_{1}\left(\sigma_{g}\right)\right)$ реализуют сопряжение исходными порождающими $\pi_{1}\left(\sigma_{g}\right)$. Эти автоморфизмы слегка отличаются от используемых нами автоморфизмов $S_{i}$ и $T_{i}$, которые были получены нами до того, как мы ознакомились с работой Цишанга [8]. В той же работе Цишанг впервые указал следующее ниже утверждение, которое является уточнением теоремы N10 из монографии [7; с. 185]. Мы приведем эту лемму с ее элементарным доказательством на основе теоремы N10.

Лемма 5. Любой элемент $\beta \in \operatorname{Aut}^{+}\left(\pi_{1}\left(\Sigma_{g}\right)\right)$ можно представить с помощью некоторого свободного автоморфизма, сохраняющего элемент $w$.

ДокАзАТЕЛЬСтво. Используя указанную теорему N10, по данному элементу $\beta \in$ $\operatorname{Aut}^{+}\left(\pi_{1}\left(\Sigma_{g}\right)\right)$ найдем представляющий его свободный автоморфизм $\gamma$, который переводит слово $w$ в некоторое слово вида $T w T^{-1}$. Пусть $\delta$ есть свободный внутренний автоморфизм, сопрягающий любой элемент $x$ группы $F_{2 g}$ с помощью слова $T^{-1}$, т.е. для любого $x \in F_{2 g}$ имеем $\delta(x)=T^{-1} x T$. Очевидно, $\delta \gamma \in N$. В силу леммы 2 найдем такой свободный автоморфизм $\delta_{1} \in N$, который на группе $\pi_{1}\left(\Sigma_{g}\right)$ действует как внутренний автоморфизм, реализующий сопряжение с помощью слова $T$. Очевидно, автоморфизм $\delta_{1}(\delta \gamma) \in N$ в группе $\operatorname{Aut}^{+}\left(\pi_{1}\left(\Sigma_{g}\right)\right)$ реализует исходный элемент $\beta$.

Лемма 5 означает, что естественное отображение $\varphi_{1}: N \rightarrow \operatorname{Aut}^{+}\left(\pi_{1}\left(\Sigma_{g}\right)\right)$ есть эпиморфизм.

Лемма 6 (Цишанг, 1982). Ядро естественного эпиморфизма $\varphi_{1}$ группы $N$ на группу $\mathrm{Aut}^{+}\left(\pi_{1}\left(\Sigma_{g}\right)\right)$ порождается элементом $\alpha$.

Ниже мы опишем в терминах порождающих и определяющих соотношений ядро естественного эпиморфизма $\varphi$ группы $N$ на группу классов отображений замкнутой поверхности рода $g \geqslant 3$.

Лемма 7. В группе $N$ выполнены соотношения

$$
\begin{gathered}
S_{i} \alpha=\alpha S_{i}, \quad T_{i} \alpha=\alpha T_{i}, \quad i=1,2, \ldots, g, \\
S_{1} T_{1} S_{1}^{\prime} T_{1}^{\prime} \ldots S_{g} T_{g} S_{g}^{\prime} T_{g}^{\prime}=\alpha^{2-2 g} .
\end{gathered}
$$

ДокАзАтельство. Соотношения (12) очевидны. Для доказательства соотношения (13) используем следующие обозначения для некоторых автоморфизмов свободной группы (ср. с (2)):

$$
\begin{aligned}
K_{i} & =S_{i} T_{i} S_{i}^{\prime} T_{i}^{\prime}, \\
W_{i} & =K_{1} K_{2} \ldots K_{i}, \quad 1 \leqslant i \leqslant g, \\
W & =W_{g} .
\end{aligned}
$$


Нам достаточно для всех порождающих $x$ группы $F_{2 g}$ доказать соотношение

$$
W_{g}(x)=w^{2 g-2} x w^{2-2 g} .
$$

Непосредственным вычислением соответствующих автоморфизмов легко проверяются соотношения

$$
\begin{aligned}
& K_{i}\left(s_{i}\right)=w^{\prime} k_{i} s_{i} k_{i}^{\prime} w, \quad K_{i}\left(t_{i}\right)=w^{\prime} k_{i} t_{i} k_{i}^{\prime} w, \\
& K_{i}\left(s_{l}\right)=w^{\prime} k_{i} w^{\prime} s_{l} w k_{i}^{\prime} w, \quad K_{i}\left(t_{l}\right)=w^{\prime} k_{i} w^{\prime} t_{l} w k_{i}^{\prime} w, \quad 1 \leqslant l<i, \\
& K_{i}\left(s_{j}\right)=w^{-2} k_{i} s_{j} k_{i}^{\prime} w^{2}, \quad K_{i}\left(t_{j}\right)=w^{-2} k_{i} t_{j} k_{i}^{\prime} w^{2}, \quad i<j \leqslant g .
\end{aligned}
$$

Учитывая, что все коммутаторы $K_{i}$ переводят слово $w$ в себя, из соотношений $(16)$ легко получаем соотношения

$$
\begin{array}{lll}
K_{i}\left(k_{i}\right)=w^{\prime} k_{i} k_{i} k_{i}^{\prime} w=w^{\prime} k_{i} w, & \\
K_{i}\left(k_{l}\right)=w^{\prime} k_{i} w^{\prime} k_{l} w k_{i}^{\prime} w, & & 1 \leqslant l<i, \\
K_{i}\left(k_{j}\right)=w^{-2} k_{i} k_{j} k_{i}^{\prime} w^{2}, & i<j \leqslant g .
\end{array}
$$

Докажем искомое соотношение (15) для порождающих $x=s_{j}$. Для порождающих $t_{j}$ вычисления будут аналогичны. Имеем

$$
\begin{aligned}
W_{g}\left(s_{g}\right) & =W_{g-1}\left(K_{g}\left(s_{g}\right)\right)=W_{g-1}\left(w^{\prime} k_{g} s_{g} k_{g}^{\prime} w\right) \\
& =W_{g-2}\left(w^{\prime} w^{-2} k_{g-1}\left(k_{g} s_{g} k_{g}^{\prime}\right) k_{g-1}^{\prime} w^{2} w\right)=\cdots \\
& =w^{-2 g+1} k_{1} k_{2} \ldots k_{g-1} k_{g} s_{g} k_{g}^{\prime} k_{g-1}^{\prime} \ldots k_{2}^{\prime} k_{1}^{\prime} w^{2 g-1}=w^{-2 g+2} s_{g} w^{2 g-2} \\
W_{g}\left(s_{g-1}\right) & =W_{g-1}\left(w^{\prime} k_{g} w^{\prime} s_{g-1} w k_{g}^{\prime} w\right)=W_{g-2}\left(K_{g-1}\left(w^{\prime} k_{g} w^{\prime} s_{g-1} w k_{g}^{\prime} w\right)\right) \\
& =W_{g-2}\left(w^{\prime}\left(w^{-2} k_{g-1} k_{g} k_{g-1}^{\prime} w^{2}\right) w^{\prime}\left(w^{\prime} k_{g-1} s_{g-1} k_{g-1}^{\prime} w\right) w\left(w^{-2} k_{g-1} k_{g}^{\prime} k_{g-1}^{\prime} w^{2}\right) w\right) \\
& =W_{g-2}\left(w^{-3} k_{g-1} k_{g} s_{g-1} k_{g}^{\prime} k_{g-1}^{\prime} w^{3}\right)=\cdots=w^{-2 g+2} s_{g-1} w^{2-2 g} .
\end{aligned}
$$

Заметим, что в (19), как и в (18), в преобразованиях $K_{j}$ при $j=g-2, g-3, \ldots, 1$ происходит последовательное сопряжение всех множителей преобразуемого слова с помощью слова $w^{-2} k_{j}$.

В общем случае для данного $i$ сначала индукцией по $j$ убедимся, что для любых $2 \leqslant i \leqslant g-1$ и $j \leqslant i$ выполнены равенства

$$
\begin{aligned}
& W_{g}\left(s_{g-i}\right)=W_{g-1}\left(w^{\prime} k_{g} w^{\prime} s_{g} k_{g}^{\prime} w\right)=W_{g-2}\left(w^{-3} k_{g-1} k_{g} w^{\prime} s_{g-i} w k_{g}^{\prime} k_{g-1}^{\prime} w^{3}\right) \\
& \quad=W_{g-2}\left(w^{\prime}\left(w^{-2} k_{g-1} k_{g} k_{g-1}^{\prime} w^{2}\right) w^{\prime}\left(w^{\prime} k_{g-1} w^{\prime} s_{g-i} w k_{g-1}^{\prime} w\right) w\left(w^{-2} k_{g-1} k_{g}^{\prime} k_{g-1}^{\prime} w^{2}\right) w\right) \\
& \quad=W_{g-2}\left(w^{-3} k_{g-1} k_{g} w^{\prime} s_{g-i} w k_{g}^{\prime} k_{g-1}^{\prime} w^{3}\right)=\ldots \\
& \quad=W_{g-j}\left(w^{-2 j+1} k_{g-j+1} \ldots k_{g-1} k_{g} w^{\prime} s_{g-i} w k_{g}^{\prime} k_{g-1}^{\prime} \ldots k_{g-j+1}^{\prime} w^{2 i-1}\right) .
\end{aligned}
$$

В частности, при $j=i$ будем иметь

$$
\begin{aligned}
W_{g}\left(s_{g-i}\right)= & \cdots=W_{g-i}\left(w^{-2 j+1} k_{g-j+1} \ldots k_{g-1} k_{g} w^{\prime} s_{g-i} w k_{g}^{\prime} k_{g-1}^{\prime} \ldots k_{g-j+1}^{\prime} w^{2 i-1}\right) \\
= & W_{g-i-1}\left(w^{-2 i+1}\left(w^{-2} k_{g-i} k_{g-i+1} \ldots k_{g-1} k_{g} k_{g-i}^{\prime} w^{2}\right)\right. \\
& \left.w^{\prime}\left(w^{\prime} k_{g-i} s_{g-i} k_{g-i}^{\prime} w\right) w\left(w^{-2} k_{g-i} k_{g}^{\prime} k_{g-1}^{\prime} \ldots k_{g-i+1}^{\prime} k_{g-i}^{\prime} w^{2} w^{2 i-1}\right)\right) \\
= & W_{g-i-1}\left(w^{-2 i-1} k_{g-i} k_{g-i+1} \ldots k_{g-1} k_{g} s_{g-i} k_{g}^{\prime} k_{g-1}^{\prime} \ldots k_{g-i+1}^{\prime} k_{g-i}^{\prime} w^{2} w^{2 i-1}\right) .
\end{aligned}
$$


При $j=g-i-1, \ldots, 1$, как и в $(19)$, каждый следующий автоморфизм $K_{j}$ будет давать сопряжение с помощью слова $w^{-2} k_{j}$. В результате получим равенства

$$
\begin{aligned}
& W_{g}\left(s_{g-i}\right)=W_{g-1}\left(w^{\prime} k_{g} s_{g-i} k_{g}^{\prime} w\right)=\ldots \\
& \quad=W_{g-i-1}\left(w^{-2 i-1} k_{g-i} k_{g-i+1} \ldots k_{g-1} k_{g} s_{g-i} k_{g}^{\prime} k_{g-1}^{\prime} \ldots k_{g-i+1}^{\prime} k_{g-i}^{\prime} w^{2} w^{2 i-1}\right)=\ldots \\
& \quad=\left(w^{-2 g+1} k_{1} k_{2} \ldots k_{g-1} k_{g} s_{g-i} k_{g}^{\prime} k_{g-1}^{\prime} \ldots k_{2}^{\prime} k_{1}^{\prime} w^{2 g-1}=w^{-2 g+2} s_{g-i} w^{2 g-2} .\right.
\end{aligned}
$$

Лемма 7 доказана.

ЛЕмма 8. Подгрупnа групnъ $N$

$$
G=\left\langle S_{1}, T_{1}, \ldots, S_{g}, T_{g}, \alpha\right\rangle
$$

является нормальной в $N$.

ДокАЗАТЕЛЬСтво. В силу леммы 3 нам достаточно доказать, что сопряжение порождающих подгруппы $G$ с помощью свободных автоморфизмов (4) переводит их в элементы из $G$. Этот факт легко проверяется непосредственным вычислением соответствующих автоморфизмов. В частности, при сопряжении автоморфизмами (4) элемент $\alpha$ переходит в себя. То же самое верно во многих других случаях. Ниже мы выпишем соответствующие результаты для всех тех случаев, когда элемент $S_{i}$ или $T_{i}$ при сопряжении с помощью той или иной порождающей (4) изменяется. Непосредственным вычислением значений автоморфизмов левой и правой частей указанных ниже равенств устанавливается, что значения этих автоморфизмов совпадают при всех значениях параметров $s_{i}$ и $t_{i}$. Результаты этих вычислений показывают, что в группе $N$ выполнены следующие соотношения:

$$
\begin{array}{rlrl}
u_{i} S_{i} u_{i}^{\prime} & =S_{i} T_{i}^{\prime}, & & \\
y_{i} T_{i} y_{i}^{\prime} & =T_{i} S_{i}, & \\
x_{i} S_{i-1} x_{i}^{\prime} & =\alpha S_{i-1} T_{i-1}^{\prime} S_{i} T_{i} S_{i}^{\prime}, & \\
x_{i} T_{i-1} x_{i}^{\prime} & =S_{i} T_{i}^{\prime} S_{i}^{\prime} T_{i-1} S_{i} T_{i} S_{i}^{\prime}, & \\
x_{i} S_{i} x_{i}^{\prime} & =\alpha^{\prime} S_{i} T_{i}^{\prime} S_{i}^{\prime} T_{i-1} S_{i}, &
\end{array}
$$

Тем самым, завершается доказательство леммы 8.

Из лемм 6 и 8 вытекает

Лемма 9. Элементы $\alpha, S_{i}, T_{i}, i=1,2, \ldots, g$, порождают ядро $\operatorname{ker} \varphi$, m.e.

$$
\operatorname{ker} \varphi=\left\langle S_{1}, T_{1}, \ldots, S_{g}, T_{g}, \alpha\right\rangle_{N}=G \text {. }
$$

Теорема 1. Ядро эпиморфизма ч группы $N$ на группу $\operatorname{Aut}^{+}\left(\pi_{1}\left(\Sigma_{g}\right)\right) / \operatorname{Inn}\left(\pi_{1}\left(\Sigma_{g}\right)\right)$ имеет следующее задание с помощью порождающих и определяющих соотношений:

$$
G=\left\langle S_{1}, T_{1}, \ldots, S_{g}, T_{g}, \alpha \mid(12),(13)\right\rangle .
$$

ДокАЗАТЕЛЬСтво. По лемме 7 соотношения (12) и (13) выполнены в $N$, а значит, и в ядре $\operatorname{ker} \varphi$. Докажем, что они дают полную систему определяющих соотношений ядра ker $\varphi$. Допустим, что в группе $N$ выполнено некоторое соотношение

$$
Z\left(S_{1}, T_{1}, \ldots, S_{g}, T_{g}, \alpha\right)=1 .
$$


Используя коммутации (12), перепишем соотношение (27) в виде

$$
Z=\alpha^{r} Y\left(S_{1}, T_{1}, \ldots, S_{g}, T_{g}\right)=1
$$

Нам нужно доказать, что соотношение (28) выводится из (12) и (13).

Из $(28)$ и $\varphi_{1}(\alpha)=1$ мы получим $\varphi_{1}(Y)=1$. Напомним, что $S_{i}$ и $T_{i}$ являются внутренними автоморфизмами $\pi_{1}$, которые сопрягают с помощью $s_{i}$ и $t_{i}$ соответственно. Отсюда следует, что $\varphi_{1}(Y)$ действует в $\pi_{1}\left(\Sigma_{g}\right)$ как сопряжение словом $y$, получаемым из $Y$ заменой заглавных букв $S_{i}$ и $T_{i}$ малыми буквами $s_{i}$ и $t_{i}$. Так как $Y$ есть тривиальный автоморфизм группы $\pi_{1}\left(\Sigma_{g}\right)$, то мы получаем, что $y=1$ в группе $\pi_{1}\left(\Sigma_{1}\right)$.

Рассмотрим группу $G_{1}$, задаваемую порождающими $S_{i}$ и $T_{i}$ и одним определяющим соотношением $W=1$. Заметим, что группа $G_{1}$ есть изоморфная копия группы $\pi_{1}\left(\Sigma_{g}\right)$, в задании которой все порождающие заменены заглавными буквами. Очевидно, в $G_{1}$ выполнено равенство $Y=1$. Согласно теории малых сокращений в результате применения в группе $G_{1}$ алгоритма Дэна к слову $Y$ получим последовательность преобразований слов вида

$$
Y \rightarrow Y_{1} \rightarrow Y_{2} \rightarrow \cdots \rightarrow 1
$$

Напомним, что применение алгоритма Дэна к несократимому циклическому слову $Y$ в группе с определяющим соотношением $W=1$ заключается в последовательной замене в $Y$ кусков циклических сдвигов слова $w^{ \pm 1}$ длины больше его половины на их дополнения в степени -1 с последующим сокращением. Очевидно, аналог этого алгоритма можно применять и в группе с определяющим соотношением (13) с той лишь разницей, что в данном случае вместо дополнения до $W^{ \pm 1}$ нужно брать это дополнение, умноженное на $\alpha^{ \pm(2-2 g)}$. При этом в результате мы получим вместо 1 некоторую степень элемента $\alpha$, скажем $\alpha^{r_{1}}$. Это означает, что только из определяющих соотношений (13) и (12) выводится равенство

$$
Y=\alpha^{r_{1}}
$$

которое, очевидно, верно и в группе $N$. Тогда в силу (28) в $N$ выполнено равенство $Z=\alpha^{\left(r+r_{1}\right)}=1$. Так как в $N$ элемент $\alpha$ имеет бесконечный порядок, то отсюда получаем $r+r_{1}=0$. Это означает, что при выводе равенства (30), используя только соотношения (13) и (12), мы фактически доказали искомое равенство (28). Теорема 1 доказана.

Заметим, что группа (26) изоморфна фундаментальной группе трехмерного многообразия Зейферта без особых слоев (см. [9; с. 200-201, формула (1) при $r=0$, формула (5) при $b=2-2 g]$ ).

Рассмотрим следующие элементы стабилизатора $N$ :

$$
r_{j}=\left\{\begin{aligned}
s_{j} & -\rightarrow s_{j+1}, \\
t_{j} & -\rightarrow t_{j+1}, \\
s_{j+1} & -\rightarrow k_{j+1}^{\prime} s_{j} k_{j+1}, \quad 1 \leqslant j \leqslant g-1 . \\
t_{j+1} & -\rightarrow k_{j+1}^{\prime} t_{j} k_{j+1},
\end{aligned}\right.
$$


Очевидно, при $|i-j|>1$ элементы $r_{i}$ и $r_{j}$ коммутируют. Легко проверить, что при $j=1,2, \ldots, g-2$ в $N$ выполнены также соотношения

$$
r_{j} r_{j+1} r_{j}=r_{j+1} r_{j} r_{j+1}
$$

Таким образом, для подгруппы $R=\left\langle r_{1}, r_{2}, \ldots, r_{g-1}\right\rangle$ группы $N$ выполнены все соотношения группы кос $B_{g}$ с $g$ нитями. Докажем, что группа $R$ изоморфна $B_{g}$.

Легко видеть, что элементы $r_{j}$ действуют на коммутаторах $k_{j}$ и $k_{j+1}$ следующим образом:

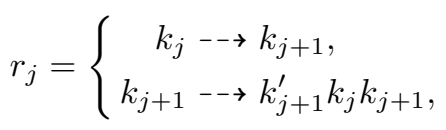

а остальные коммутаторы $k_{i}$ оставляют на месте.

Отсюда следует, что каждый элемент $r_{j}$ отображает подгруппу группы $F_{2 g}$

$$
K=\left\langle k_{1}, k_{2}, \ldots, k_{g}\right\rangle \cong F_{g}
$$

на себя и это отображение есть автоморфизм. Таким образом, мы имеем естественный гомоморфизм $\theta: R \rightarrow \operatorname{Aut}\left(F_{g}\right)$.

Согласно теореме N6 (см. [7; c. 182]) элементы $\theta\left(r_{j}\right) \in \operatorname{Aut}\left(F_{g}\right)$ порождают группу $\theta(R)$, изоморфную группе кос $B_{g}$. Как было показано выше, все соотношения группы $\theta(R) \cong B_{g}$ уже выполнены в $R$. Следовательно, группа $R$ также изоморфна $B_{g}$.

Теорема 2. Указанная выше подгруппа $R$, изоморфная группе кос $B_{g}$, в результате абелизации свободной группы $F_{2 g}$ отображается на подгруппу группы Вайля для $\operatorname{Sp}(2 g, \mathbb{Z})$, порожденную всеми матрицами, не содержащими -1.

ДокАЗАтЕЛЬСтво. Хорошо известно (см. [7; с. 187]), что при абелизации группы $F_{2 g}$ естественное отображение $\psi: \operatorname{Aut}\left(F_{2 g}\right) \rightarrow \operatorname{GL}(2 g, \mathbb{Z})$ индуцирует отображение подгруппы $N$

$$
N \stackrel{\psi}{\rightarrow} \mathrm{Sp}(2 g \mathbb{Z})
$$

При этом выписанные выше автоморфизмы (31) переходят в матрицы $\psi\left(r_{j}\right)$, получающиеся из единичной матрицы заменой диагонального блока размера 4 от строки $2 j-1$ до строки $2(j+1)$ на следующий блок:

$$
\left(\begin{array}{llll}
0 & 0 & 1 & 0 \\
0 & 0 & 0 & 1 \\
1 & 0 & 0 & 0 \\
0 & 1 & 0 & 0
\end{array}\right) .
$$

Подгруппа, порожденная всеми матрицами $\psi\left(r_{j}\right)$, изоморфна группе перестановок $S_{g}$ и имеет индекс $2^{g}$ в группе Вайля для $\operatorname{Sp}(2 g, \mathbb{Z})$. Очевидно, все матрицы этой подгруппы составлены только из нулей и единиц.

Таким образом, мы нашли в стабилизаторе $N$ подгруппу, изоморфную группе кос $B_{g}$, которая в отображении (33) является прообразом подгруппы группы Вайля, порожденной матрицами, не содержащими -1. 


\section{СПИСОК ЦИТИРОВАННОЙ ЛИТЕРАТУРЫ}

[1] M. Dehn, "Die Gruppe der Abbildungsklassen", Acta Math., 69 (1938), 135-206.

$[2]$ W. B. R. Lickorish, "A finite set of generators for the homeotopy group of a 2-manifold", Math. Proc. Cambridge Philos. Soc., 60 (1964), 769-778; "Corrigendum: On the homeotopy group of a 2-manifold", Proc. Cambridge Philos. Soc., 62 (1966), 679-681.

[3] S. P. Humphries, "Generators for the mapping class group", Topology of Low-Dimensional Manifolds, Proc. Second Sussex Conf. (Chelwood Gate, 1977), Lecture Notes in Math., 722, Springer, Berlin, 1979, 44-47.

[4] A. Hatcher, W. Thurston, "A presentation for the mapping class group of a closed orientable surface", Topology, 19:3 (1980), 221-237.

[5] B. Wajnryb, "An elementary approach to the mapping class group of a surface", Geom. Topol., 3 (1999), 405-466.

[6] Х. Цишанг, Э. Фогт, Х.-Д. Колдевай, Поверхности и разрывные группы, Наука, М., 1988.

[7] В. Магнус, А. Каррас, Д. Солитэр, Комбинаторная теория групn, Наука, М., 1974.

[8] H. Zieschang, "A note on the mapping class groups of surfaces and planar discontinuous groups", Low-Dimensional Topology (Chelwood Gate, 1982), London Math. Soc. Lecture Note Ser., 95, Cambridge Univ. Press,, Cambridge, 1985, 206-213.

[9] H. Seifert, "Topologie dreidimensionaler gefaserter Räume", Acta Math., 60 (1933), $147-238$.

С. И. Адян

Поступило

Математический институт им. В. А. Стеклова

11.07 .2006

E-mail: sia@mi.ras.ru

\section{Ф. Груневальд}

Mathematishes Institut der Heinrich-Heine-Universität Düsseldorf

E-mail: fritz@math.uni-duesseldorf.de

Й. Меннике

Fakultät für Mathematik der Universität Bielefeld

E-mail: mennicke@math.uni-beilefeld.de

\section{А. Л. Таламбуца}

Математический институт им. В. А. Стеклова

E-mail: altal@mi.ras.ru 